Scientific Visualization, 2020, volume 12, number 3, pages 38 - 50, DOI: 10.26583/sv.12.3.04

\title{
Spin Diode Based Microwave Registration and Holographic Visualization of Wave Front Scattering for an Autonomous Driving System
}

\author{
K.A. Zvezdin'1,A,B,C, D.R. Leshchiner²,A,C, A.F. Popkov3,A, P.N. Skirdkov4,A,B,C, \\ A.G. Buzdakov5,A, G.N. Chepkov6,A \\ A Moscow Institute of Physics and Technology (MIPT) \\ B Prokhorov General Physics Institute of the Russian Academy of Sciences \\ c Russian Quantum Center (RQC) \\ ${ }^{1}$ ORCID: 0000-0002-9050-7782, konstantin.zvezdin@gmail.com \\ 2 ORCID: 0000-0002-7881-3816, dmitry 1111@inbox.ru \\ 3 ORCID: 0000-0002-9583-0941, afpopkov@inbox.ru \\ 4 ORCID: 0000-0003-1782-5044, petr.skirdkov@phystech.edu \\ 5 ORCID: 0000-0002-7264-314X, aleksandr.buzdakov@phystech.edu \\ 6 ORCID: 0000-0001-8937-5586, chepkov@phystech.edu
}

\begin{abstract}
In the paper, we discuss the scheme and the prospects of holographic visualization for microwave scattering objects. At the core of our method is microwave registrationbased on spin diodes, to identify obstacles to the movement for the car's autonomous driving system. The resolution of the holographic system grows with wavelength decrease. However, the recording capacity at a given level of irradiating signal and diode noise decreases. We give an algorithm for the numerical reconstruction and visualization of obstacles. Estimates for the resolution and the reliability of object identification, depending on the distance to the obstacle, obtained by numerical modeling. We show that there is an optimal wavelength to reach the maximum distance of microwave recording, taking into account the system resolution and the acceptable signal level. Highly sensitive spin diodes, feasible for the $2-30 \mathrm{~cm}$ wavelength range, nearly approach the optimum range, which is $\sim 0.5^{-1} \mathrm{~cm}$ for Schottky diodes, in their holographic visualization ability. Based on the Kotelnikov theorem for the sampling frequency of a harmonic signal, and on numerical experiments, the requirements for the placement density of the receiving elements of the recorder antenna array were determined depending on the distance to the object, the number of sensors and the recording window size. We show that a microwave-recording device based on spin diodes can be promising for an autonomous driving system in conditions of constrained movement with poor visibility and high noise.
\end{abstract}

Keywords: microwave radiation, holographic registration, autonomous driving system, spin diode, detection range.

\section{Introduction}

The design of vehicle's surroundings monitoring tools is a problem of great significance in area of modern vehicles development and their control automation. One of the essential safety characteristics of the system is its ability to detect objects and monitor surroundings in adverse conditions - in presence of fog, snow or rain, in night lighting, at traffic overload or while parking in a difficult environment. Leading manufacturers have proposed various solutions to this problem. Optical surveillance cameras work effectively only in good light conditions. Night vision systems operating in the IR range depend significantly on weather condi- 
tions: active ones on the presence of rain, fog or snow, passive ones on the temperature of objects in comparison to the environment. Sound vision systems (parking sensors) do not work well in conditions of heavy rain, noise, or a detection range of more than $3 \mathrm{~m}$. For adequate and timely decision-making, some more detailed information on the detected objects is also required. In conditions of poor visibility, microwave vision devices are ones to prefer - centimeter-wave microwave radars are suitable for distant object detection even in bad weather conditions. There has recently [1-2] been shown the feasibility of holographic image registration in the microwave range using a Wi-Fi emitter. In this work, we study the potential of the method of holographic detection and visualization of objects using highly sensitive spin diodes. Those diodes possess advantages at low microwave irradiation power [3-6, 20-22] and can show a significantly higher sensitivity than the sensitivity of a Schottky diode at a similar level of equivalent input noise [16]. Estimates show that for a specified sensitivity, taking into account the radiation safety standards, there exists at least $25 \mathrm{~m}$ safe obstacle detection range. As it is well known, one of the key requirements for automotive recognition systems is an ability to stop the car upon the obstacle detection, i.e. registration of obstacles at a safe distance. While manually driving a car at a speed of $48 \mathrm{~km} / \mathrm{h}$, safely making a decision requires a distance to the object of about $23 \mathrm{~m}$ on a dry surface [7]. In bad weather conditions, it increases to $80 \mathrm{~m}$. However, in the case of a fully automated car in good technical condition, if driving at a speed of $30 \mathrm{~km} / \mathrm{h}$, the braking capabilities provide a stop on dry surfaces at a distance of 6 meters or less, and a decrease in the collision speed to prevent injuries even at shorter distances. Thus, for a fully automated car in congested urban traffic conditions, in a traffic zone with a speed limit of $30 \mathrm{~km} / \mathrm{h}$, or while parking, the required level of security even at poor visibility and noise would be achieved when recognizing obstacles at a range of up to 5-10 $\mathrm{m}$ with data processing time less than $0.1 \mathrm{sec}$.

The holographic method of microwave visualization of images based on spin diodes has been previously under discussion regarding mainly near-field registration. The use of spin diodes in a device that does microwave visualization of objects for an autonomous driving system, as far as we know, was not under discussion before. Prior to moving on to methods of object recognition in microwave imaging with spin diodes, we discuss the requirements on the parameters of a recognition system in an autonomous driving environment.

\section{System Requirements and Sensor Configuration}

The holographic method of optical registration of images is well known [8] and is widely used for visualization of various objects $[9,10]$. The possibility of expanding the range of holographic visualization of images to the terahertz range using the holographic principle of recording the wave front using a high-resolution video camera pointed out, for example, in [11]. The earlier proposed holographic vision systems assumed broadband multi-position irradiation with an excess density of receiving elements. Unlike the photodetector matrix of an optical video recorder, a microwave holographic system based on spin diodes not only allows you to obtain information about both the amplitude and phase of the received wave front, but also makes it possible to use the spin diode as a microwave mixer, which simplifies the microwave recording system [4-6]. The frequency at which the sensitivity of the spin diode is at an acceptable level (see requirements for diodes below) can reach $10 \mathrm{GHz}$ or higher, which corresponds to a wavelength of $\lambda=3 \mathrm{~cm}$ or less. 


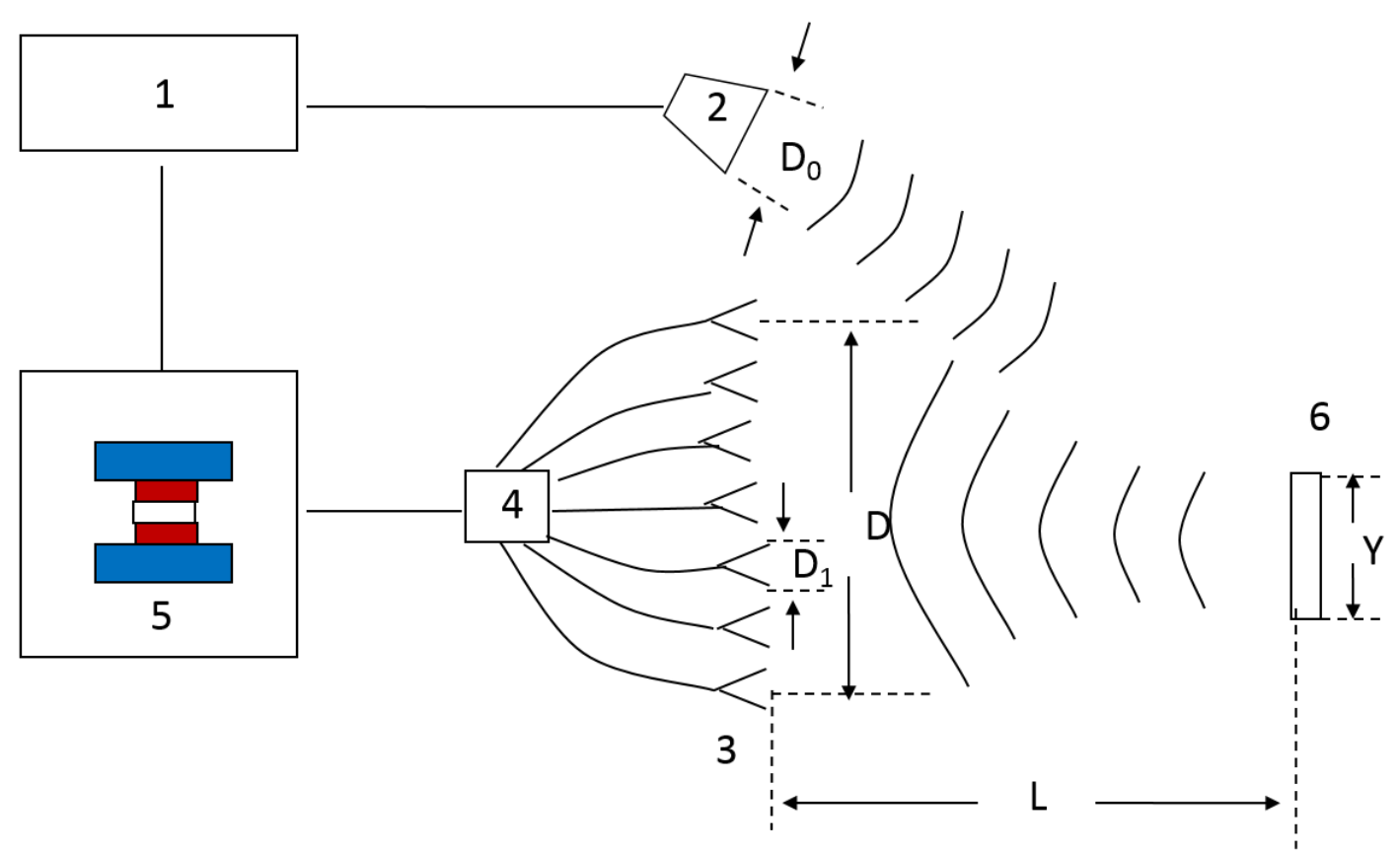

Fig. 1. Microwave holographic registration scheme based on a spin diode:

1 - microwave generator, 2 - horn antenna, 3 - antenna array, 4 - channel selector, 5 - recording unit with a spin diode, 6 - registered object

According to Abbe's classical theory of optical resolution for monochromatic irradiation, see e.g. [12], the detection range is determined by the diffraction limit $L=\frac{Y r_{n}}{0.77 \lambda}$, where $\mathrm{Y}$ is the width of the object, $r_{n}=D / 2$ is the radius of the outer Fresnel zone, equal to the radius of the reception window of the recorder. Therefore, for example, for an object with a width of $\mathrm{Y}=30$ $\mathrm{cm}$ with a holographic recorder diameter of $2 \mathrm{~m}$ at a wavelength of $3 \mathrm{~cm}$, we get $\mathrm{L}=13 \mathrm{~m}$. The requirement for the density of the sensors along the diameter of the recorder is defined by Kotelnikov's theorem. According to this theorem, accurate signal reproduction is given when sampling a signal with a resolution of at least a half-period of the maximum spatial frequency of the amplitude and phase modulation of the wave front. The discreteness of the reception of the wave front can be associated with the discreteness of the zones of the zone plate. The minimum width of the Fresnel zone at the edge of the zone plate sets the maximum frequency of the wave front from the point image. Therefore, in accordance with the Kotelnikov theorem, this width determines the maximum step distance of the signal sampling. The width of the outer Fresnel zone is given by the approximate formula

$$
\Delta \mathbf{r}_{\mathbf{n}}=\mathbf{r}_{\mathbf{n}+1}-\mathbf{r}_{\mathbf{n}} \approx \frac{\lambda \mathbf{L}}{2 \mathbf{r}_{\mathbf{n}}}
$$

where $r_{n}=\sqrt{\lambda L n}$ is the radius of the $\mathrm{n}$-th (outer) Fresnel zone.

This shows that the resolution for the registration of the wave front could be much lower than the step in the usual registration of the hologram, which should be of the order of half the wavelength. For example, at a distance of $12 \mathrm{~m}$, a radius of $1 \mathrm{~m}$, and a wavelength of $3 \mathrm{~cm}$, the width of the zone is $\Delta r_{n}=18 \mathrm{~cm}$. In the latter case, 14 sensors are sufficient for a diameter of 2 m.

\section{Image restoration and visualization technique}

The received coherent wave front within the window of the holographic recorder is used to obtain an image in a given window and for further numerical processing. In this regard, the 
task of recognizing objects includes separating the signal from interfering noise and restoring the shape of the initial distribution of scattering density. The restoration of the initial distribution may use the inverted matrixes of the convolution kernel of the direct image (the inverse problem of image restoration). In the case of a noisy signal, the resolution and detection range will depend on the effectiveness of the signal extraction algorithm.

We consider the following form of the image restoration problem. Given are the positions of one monochromatic radiation source and several receiving sensors. The formula for calculating the scattered signals (the values getting both their phase and amplitude) $-P(p)$ given by:

$$
\int f(\bar{r}) \frac{g(\bar{r})}{R} e^{-i k R} d \bar{r}=P(p)
$$

There $f(\bar{r})$ is the value of the scattering density at the point $\bar{r}$ of the image recovery region; $g(\bar{r})$ is the radiation intensity in the direction $\bar{r} ; p$ is the position of the sensor. $\omega$ is the signal frequency; $R$ is the length of the broken line that connects the source position to the point $\bar{r}$, and then to the position of sensor; $k=\frac{2 \pi}{\lambda}$ is the wave vector and $\lambda=6 \mathrm{~cm}$ is the radiation wavelength.

Our algorithm to reconstruct the scattering density field from the holographic scattering pattern is based on solving the inverse problem in Fresnel - Kirchhoff integral representation with consideration for the amplitude-phase modulation of the wave front by the scattering object and it was first described in $[13,14]$. To improve the stability of the solution for the inverse scattering problem, the Tikhonov regularization algorithm was applied [15] in the Fourier space for the harmonics of both the microwave field and the kernel of the inverse scattering problem, as well as the threshold-based signal reconstruction algorithm. The algorithm is robust against computational errors and the presence of random noise; it is efficient in the situation of a small number of sensors and an object irradiation field restricted to a single emitter.

The restoration made for the scattering density breaks into two successive separate stages, different both in substantial motivation and in solution technology. At the first stage, we restore the assumed suitable form of the scattering density field, described by the sum of loworder Fourier harmonics, ensuring the smoothness of this solution. Suppression of high harmonics made by a suitable choice of the Tikhonov regularizing matrix. The second stage, on the contrary, is aimed at finding the shape of the restored density in the form of a piecewise constant function with predefined fixed local density values (i.e., already known typically encountered values of the scattering density of known materials). Clustering, by assigning clusters with threshold cutoff, the local density values restored at the first stage around predefined density values achieves that. Such a two-stage regularization scheme (based on the Tikhonov matrix at the first stage and local clustering of density values at the second) allows one to achieve high resistance to signal noise even at a low number of receiving channels (antenna array sensors). At the second stage, the form of the problem solution defines a scene built of bounded objects of a given density. This is exactly the representation required by the scene visualization task. However, both the initial regularization of the solution and the noise suppression are much more effective in the Fourier space.

The density reconstruction and the regularization with the high harmonics suppression at the first stage of the algorithm is as follows. The integral equation (2) for $f(\bar{r})$ in the space of Fourier harmonics of given orders is a linear equation, the kernel of it given by a fixed finite ma$\operatorname{trix} A$. Accordingly, its solution might proceed by applying the inverse matrix $A^{-1}: f(\bar{r})=$ $A^{-1} P(p)$. However, the naive form of the matrix $A^{-1}$ does not exist, since $A$ is, generally speaking, a rectangular matrix, and, practically, the size of the vector $f(\bar{r})$ (the number of harmonics) is significantly larger than the size of the vector $P(p)$ (the number of sensors). That is, the system is underdetermined. With that, the solution with Tikhonov regularization proceeds by substituting the (non-existent) matrix $A^{-1}$ with the matrix $\left(A^{T} A+\Gamma^{T} \Gamma\right)^{-1} A^{T}$, where $A^{T}$ and $\Gamma^{T}$ are Hermitian conjugates to $A$ and $\Gamma$. The matrix $\Gamma$ is a Tikhonov regularizing matrix (a matrix for a quadratic form in the space of solutions) - the choice of which is ours 
and must depend on the essential formulation of the regularization problem. In our case (suppression of high harmonics), the suitable form for $\Gamma$ is a diagonal matrix, the weights in its diagonal being proportional to the product of the orders of the corresponding harmonics for each of the spatial coordinates. This way, optimal suppression is ensured for high order harmonics - the experiments showed that using, say, the product of harmonics orders in degrees higher than the first gives too much suppression of high harmonics with a deterioration of reproduction quality for the scattering object shape and its visualization. Alternatively, an excessively weak suppression of high harmonics leads to the same.

The task of clustering local density values around given known values is a one-dimensional clustering problem. It is solvable by many standard methods; one of the simplest is the threshold cutoff at the point of the lowest local density of values within the interval - we used it successfully here to get a very adequate reconstruction of the external shape of the object both to visualize it and to apply for the task of recognizing obstacles.

\section{Simulation results and detection confidence limits}

To assess the effectiveness of the objects visualization algorithm we developed, we carried series of computer simulation numerical experiments for model problems detecting a number of three-dimensional, two-dimensional and one-dimensional objects, for a more detailed study of the algorithm behavior for various parameters and of the theoretical capacities of the method.

Below are the examples of visualization of three-dimensional obstacles in the frontal plane of the XY projection - the contours of the reconstructed image as shown in comparison with the contour of the original object. The virtual model of the experiment involved the following parameters: distance to the object, rotation of the object, position of the object along the scene's width, the direction of the radiation emitter, radiation scattering pattern, and configuration of the array of sensors. Series of experiments made it possible to establish the boundaries of applicability of the system.

For the job, we chose two distinct objects, that is, three-dimensional densities $f(\bar{r})$, which we then projected onto the XY plane. The first object had a simpler form than the second one. We chose the cube as the first object (Fig. 2a). As the second one (Fig. 2b) we chose a human figure.
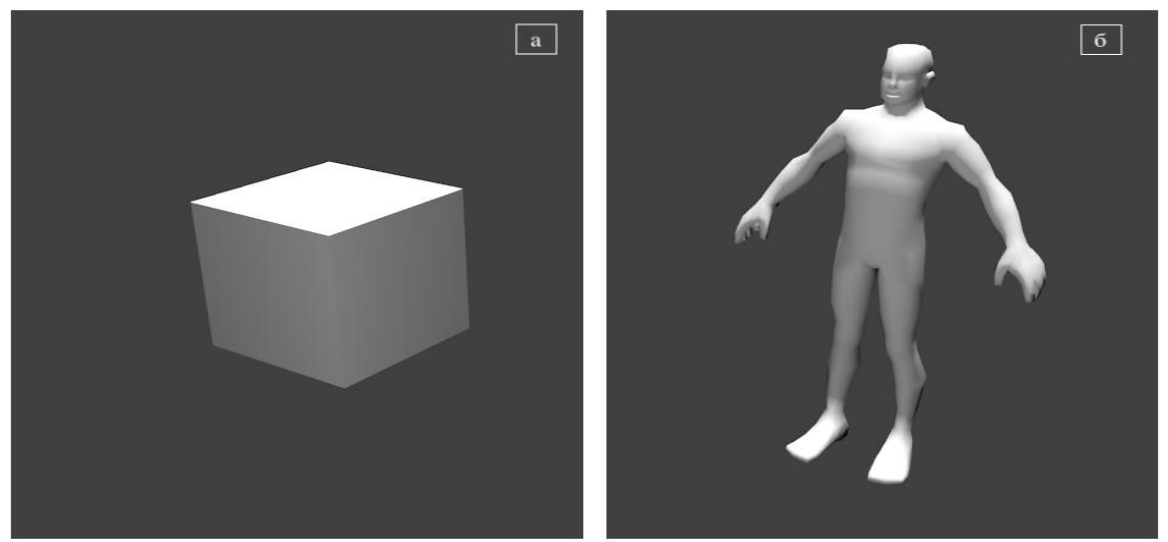

Fig. 2: Densities used in the simulation ( $\mathrm{a}$ - density in the shape of a cube, b - density in the shape of a male human figure).

To apply the proposed algorithm, we had to calculate the signals gotten from the receiving elements. The emitter wavelength: $\lambda=6 \mathrm{~cm}$, the sensor array 13x13, the distance between the sensors $20 \mathrm{~cm}$. After the numerical calculation of the integrals (formula 2), we obtained the phase and amplitude values on each of the sensors. The figures below demonstrate an example of the obtained values of phases and amplitudes. Fig. 4 shows data for an object in the form of a cube located at a distance of $360 \mathrm{~cm}$ from the holographic stand (Fig. 3a). Fig. 5 
shows similar data for an object in the form of a man (Fig. 3b) located at the same distance from the stand.
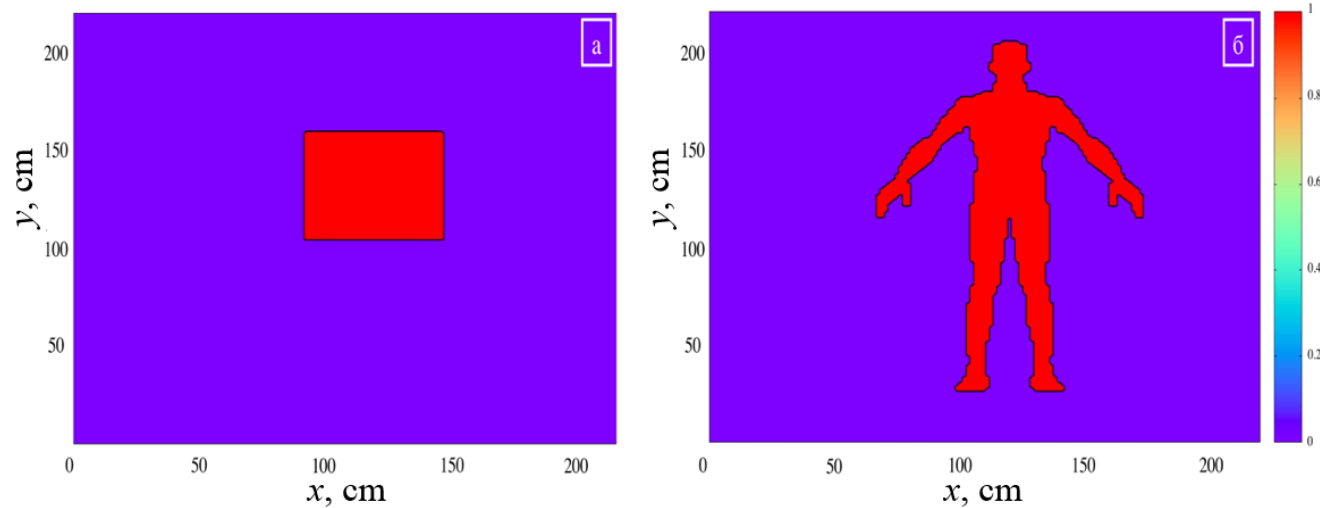

Fig. 3: Projection of density in the shape of a cube (a) and of a man (b) on a plane.
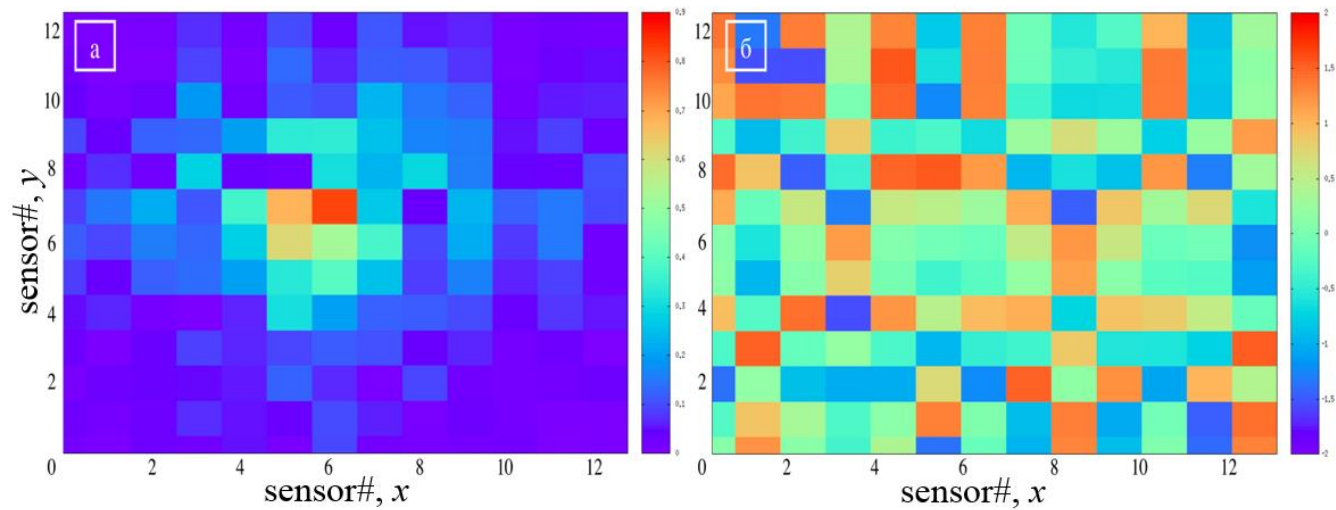

Fig. 4: Example of received signals for the density of a cube (a - amplitude, b - phase).
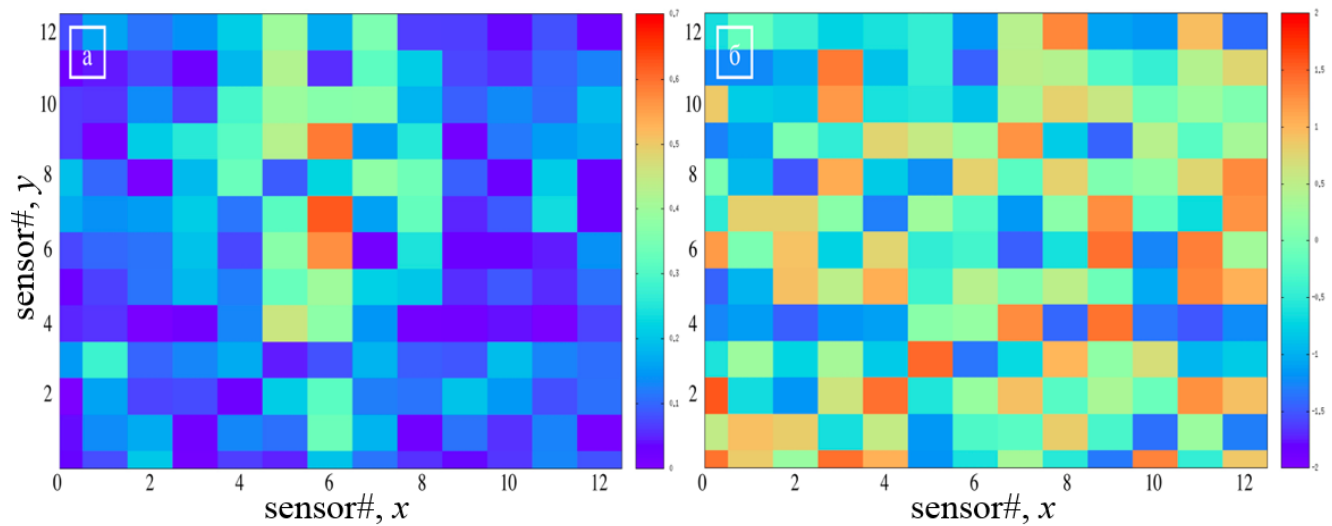

Fig. 5: Example of received signals for the density in the human shape (a - amplitude, b phase).

Then, upon getting the signals at the receiving sensors, the proposed restoration algorithm is applied. Figure 6a and Figure 6b show examples of reconstructed two-dimensional densities. 

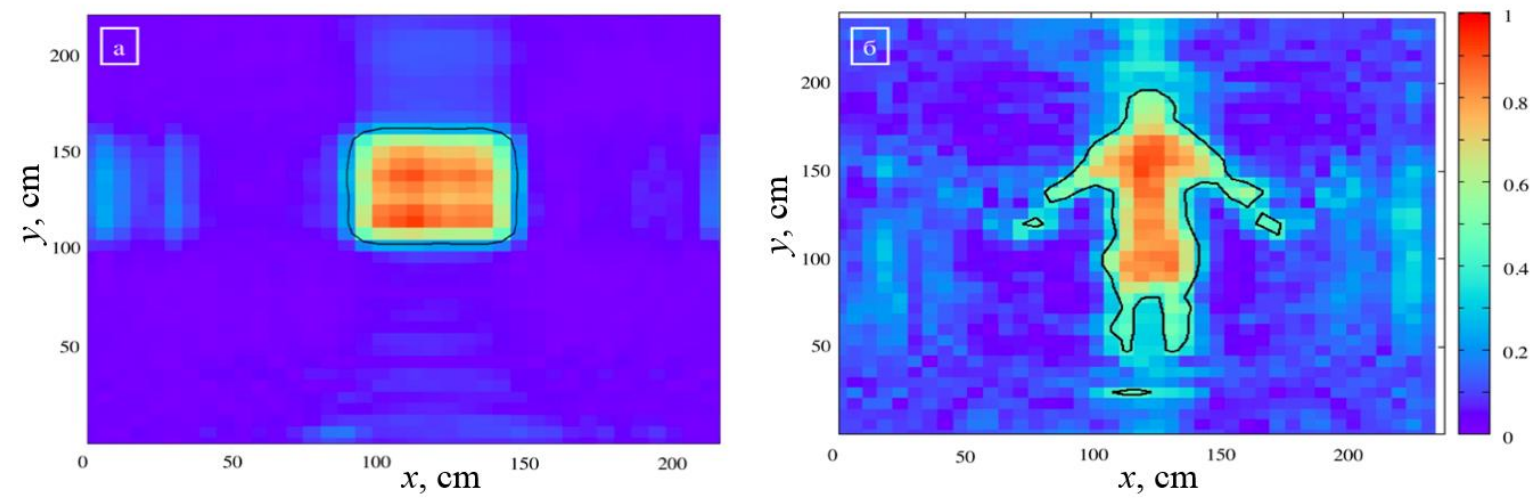

Fig. 6: Examples of reconstructed densities (a - density in the shape of a cube, b - density in the shape of a man).

\subsection{Algorithm quality assessment.}

\subsubsection{Dependence of the visualization quality on the distance from the object to the emitter and on the position of the object.}

We used these three indicators to assess the quality of visualization: precision (accuracy), recall (completeness), F1-measure. Recall definition was the ratio of the area of the correctly identified part of the initial density to the area of the initial density; precision definition was the ratio of the area of the correctly identified part to the area of the entire reconstructed contour. The F1 measure defined as the harmonic mean of recall and precision:

$$
F 1=\frac{2 * \text { precision } * \text { recall }}{\text { precision }+ \text { recall }}
$$

For both objects presented above, we obtained the dependencies of the F1 measure on the distance from the emitter to the object and the horizontal position of the center of mass of the object (see Fig. 7 and Fig. 8). Figure 7 shows the dependence of the F1 measure (indicated by color, on the scale given on the right) for the density in the shape of a cube, and Figure 8 shows the dependence for the density in the shape of a man. It is evident there that an acceptable recognition quality is maintained up to $20 \mathrm{~m}$ - which is consistent with the stated goals for detecting road obstacles in the near distance range.

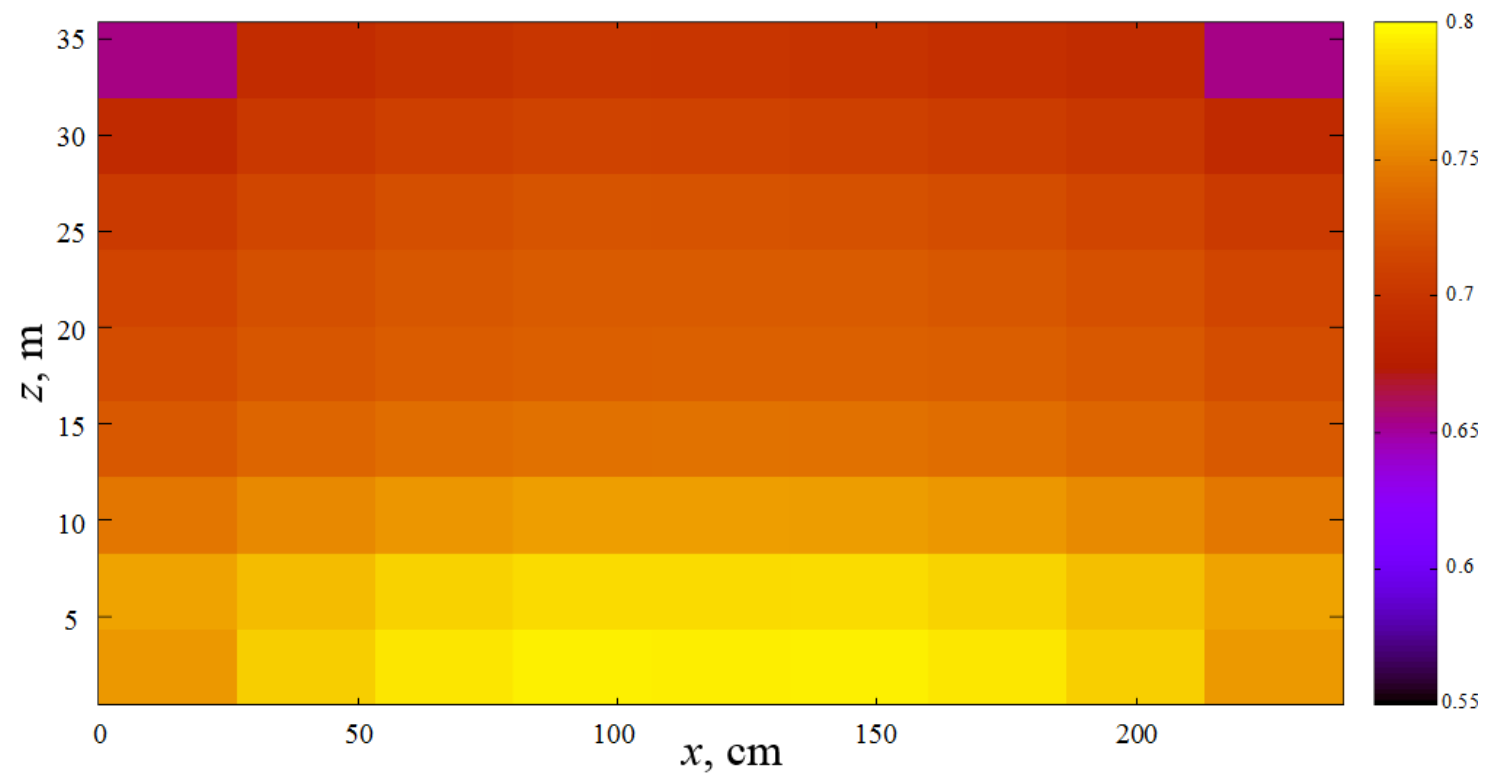

Fig. 7: F1-measure dependence for the density in the shape of a cube (the $x$-axis defines the vertical position of the object; the $z$-axis defines the distance from the sensors to the object). 


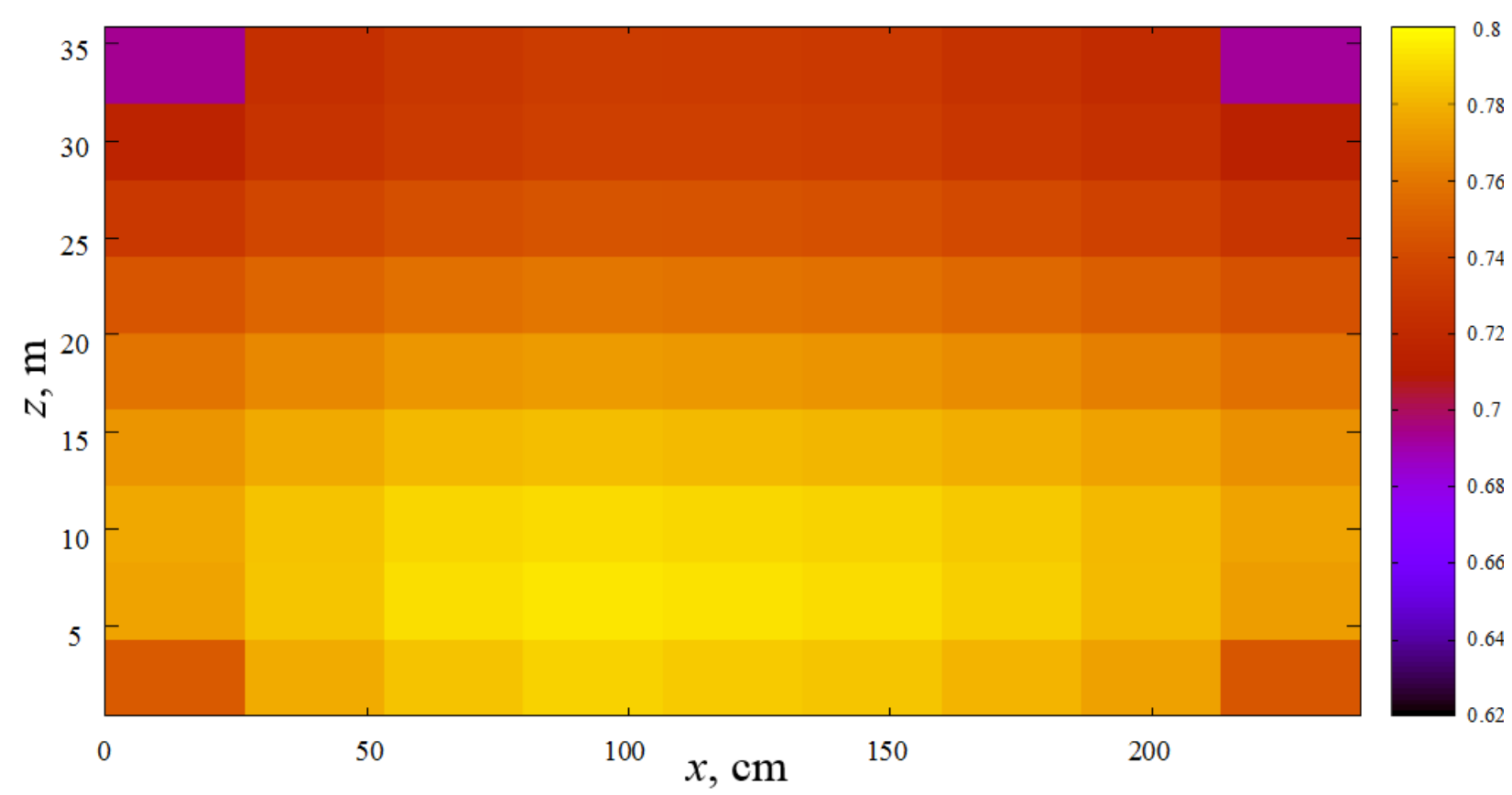

Fig. 8: F1-measure dependence for the density in the shape of a man (the $x$-axis defines the vertical position of the object; the $z$-axis defines the distance from the sensors to the object).

\subsubsection{The dependence of the recognition quality on noise.}

Another parameter for evaluating the quality of the algorithm was noise tolerance. The noise added as follows - independent normally distributed random variables added to the signal of each sensor calculated by the formula (2). The noise level measured in fraction of the mean square amplitude of the signal over the entire field of the sensors. Using this, we obtained the dependence of the immunity of the algorithm to the noise, on the object distance. Below is a plot of the maximum noise, at which there is still an opportunity to detect the object, in dependence of the distance. Here we used no formal detection criteria, assessing the detectability "by eye".

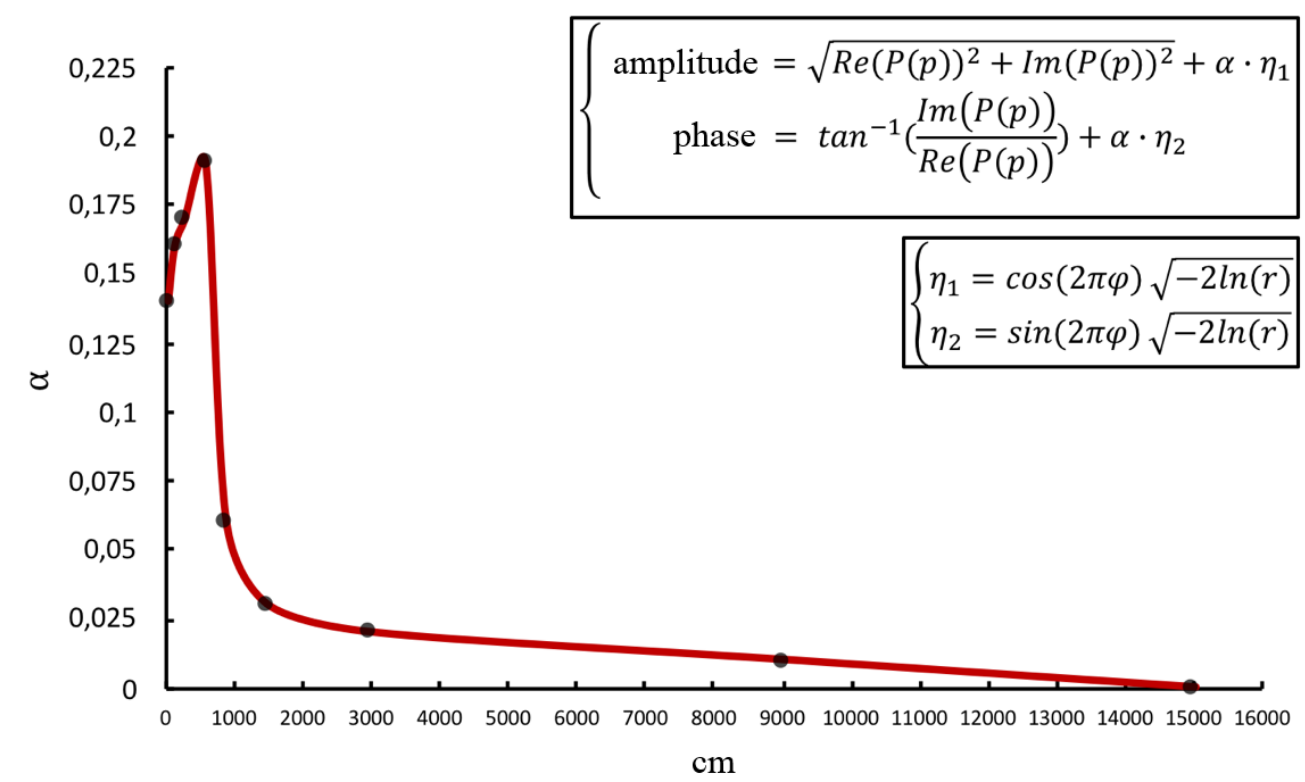

Fig. 9: Dependence of the maximum permissible noise on the distance to the object. 
From this data, the following conclusions can be drawn: an optimal distance with respect to noise tolerance is up to $6 \mathrm{~m}$; noise tolerance is quite high: a practically acceptable resistance level is maintained up to $20 \mathrm{~m}$.

\subsubsection{The dependence of the recognition error probability on the distance - for two strip reflectors.}

The simulation results show that the resolution efficiency (i.e., the minimal resolvable strip gap width) for one-dimensional objects in the form of two strip reflectors depends on the distance as $Y=\kappa \lambda L / 2 r_{n}$, where $\kappa=Y / \Delta r_{n}(\mathrm{n}=2)$ is the ratio of the gap width to the size of the Fresnel zone. Table 1 below shows the probability of recognition error for a given distance to the object and the number of receiver sensors. The probability of error defined as the percentage of cases with resolution of the objects not achieved, out of all checked combinations of object parameters (reflector width, gap width) satisfying the above resolution conditions (limits for $Y$ ).

Table 1. The dependence of the probability of recognition error on the distance $(L)$ to the object and on the number of sensors $\left(N_{S}\right)$ in the line of receivers at a reception window width of $2 \mathrm{~m}$.

\begin{tabular}{|l|l|l|l|l|l|}
\hline $\mathrm{L} \backslash \mathrm{N}_{\mathrm{S}}$ & 4 & 8 & 16 & 32 & 64 \\
\hline $\mathrm{O} .3 \mathrm{~m}$ & --- & $18.1 \%$ & $1.7 \%$ & $0.2 \%$ & $0.0 \%$ \\
\hline $0.45 \mathrm{~m}$ & $13.3 \%$ & $27.4 \%$ & $5.3 \%$ & $2.6 \%$ & $2.2 \%$ \\
\hline $0.6 \mathrm{~m}$ & $31.4 \%$ & $4.8 \%$ & $5.1 \%$ & $4.8 \%$ & $3.4 \%$ \\
\hline $1.5 \mathrm{~m}$ & $0.0 \%$ & $9.7 \%$ & $1.9 \%$ & $1.3 \%$ & $2.9 \%$ \\
\hline $3 \mathrm{~m}$ & $24.0 \%$ & $17.6 \%$ & $4.2 \%$ & $4.3 \%$ & $3.0 \%$ \\
\hline $4.5 \mathrm{~m}$ & $8.3 \%$ & $16.7 \%$ & $5.6 \%$ & $6.3 \%$ & $6.3 \%$ \\
\hline $6 \mathrm{~m}$ & --- & $30.0 \%$ & $11.6 \%$ & $9.5 \%$ & $7.4 \%$ \\
\hline $15 \mathrm{~m}$ & --- & $20.0 \%$ & $20.0 \%$ & $53.3 \%$ & $53.3 \%$ \\
\hline
\end{tabular}

It follows from the table that the most reliable recognition conditions for a wavelength of 3 $\mathrm{cm}$ and a reception window of $2 \mathrm{~m}$, for an object $40 \mathrm{~cm}$ wide, are: the distance to the object not more than $6 \mathrm{~m}$, and the number of receiving antenna sensors along the width of the reception window of at least 16 .

\section{Irradiation safety requirements and detection range}

In addition to the conditions of optical resolution, the sensitivity and the level of the equivalent input noise of the detecting device also affect the detection range. Let us consider these conditions for a spin diode, which can demonstrate the sensitivity exceeding the sensitivity of a Schottky diode at a similar level of equivalent input noise [16]. The decrease in power coming to the receiving antenna, at the angle of the horn antenna $\theta=\frac{\lambda}{D_{0}}$, the size of the reflecting mirror $Y$, the distance to the mirror object $L$ and the width of the receiving detector $D_{1}$, one may approximately estimate by the formula

$$
\frac{P_{1}}{P_{2}}=\left(\frac{Y D_{0}}{L \lambda} \cdot \frac{L D_{1}}{2 Y L}\right)^{2}=\left(\frac{D_{0} D_{1}}{2 L \lambda}\right)^{2}
$$

The safe dose of volumetric exposure for humans is, according to the published data, $I_{G}=0.08 \mathrm{~W} / \mathrm{kg}[16]$. Therefore, with a weight of $G=50 \mathrm{~kg}$ and an irradiated frontal surface of a person of approximately $S=1 \mathrm{~m}^{2}$, we obtain the intensity of safe exposure $I_{S}=\frac{I_{G} G}{\mathrm{~S}}=$ $4 \mathrm{~W} / \mathrm{m}^{2}$. This threshold intensity is created by a horn antenna at a distance of $R=1 \mathrm{~m}$ with a source of power $P_{0}=\frac{\pi}{2} I_{S} R^{2} \sim 6 \mathrm{~W}$. On the other hand, the signal detection range is determined by the relation $P_{1}=N P_{N}$, where $N$ is the signal-to-noise ratio, $P_{N}=V_{N} / \varepsilon$ is the input threshold sensitivity of the spin diode, which is proportional to the noise level of the diode voltage 
$V_{N}$ and inversely proportional to its sensitivity $\varepsilon=\frac{d V}{d P}$. Assuming that the wavelength determines the useful area of the microwave source and receiver, i.e. $D_{1}^{2} D_{2}^{2} \sim \eta \lambda^{4}$, where the parameter ${ }^{\eta}$ takes into account the utility coefficient of the receiving antenna, the efficiency of the transmitting antenna and the matching of the receiving path, from the formula (3) we find

$$
L=\left(\frac{\pi \eta I_{s} R^{2}}{8 N P_{N}}\right)^{\frac{1}{2}} \cdot \lambda
$$

For the noise equivalent power of a spin diode $P_{N} /(\Delta f)^{1 / 2}=4 \cdot 10^{-12} \mathrm{~W} / \mathrm{Hz}^{1 / 2}[16]$, for the

maximum sensitivity $\varepsilon \sim 10^{4} \mathrm{mV} / \mathrm{mW}$, achieved with a bias current, the noise threshold power with a bandwidth $\Delta f=10 \mathrm{kHz}$ is $P_{N}=4 \cdot 10^{-10} \mathrm{~W}$. For estimates, we set $\eta=0.1, N=4, R=1$ $\mathrm{m}, \lambda=3 \mathrm{~cm}$, then for the adopted noise level of the spin diode we obtain the detection range $L$ $=250 \mathrm{~m}$. For a Schottky diode, with a noise equivalent power $P_{N} /(\Delta f)^{1 / 2}=2 \cdot 10^{-12} \mathrm{~W} / \mathrm{Hz}^{1 / 2}$,

we get roughly the same estimate for the threshold noise power.

In the absence of a bias current, the sensitivity $\varepsilon$ of the spin diode decreases by a factor of 100 or more. In the latter case, the range of safe detection (with the accepted range of safe exposure $R=1 \mathrm{~m}$ ), according to estimates based on formula (4), will be about $L=25 \mathrm{~m}$. With a detection range of up to $10 \mathrm{~m}$, the input power of the generator should be at least $P_{0} \sim 2 W$. The irradiation safety requirements definitely met here.

\section{Microwave properties of spin diodes and optimal wave- length}

The spin diode is a magnetic tunnel junction with a high magnetoresistance. As a result of the non-linearity of the current-voltage characteristic, the constant voltage occurs at the junction with a microwave change in the tunnel current. The effect of rectifying a microwave signal by a spin diode has several mechanisms based on current-induced ferromagnetic resonance, thermal transfer of the spin (spin Seebeck effect), and modulation of perpendicular anisotropy induced by the voltage at the junction $[4,16,17]$. Other physical mechanisms of spin systems response discussed in $[18,19]$. Spin diodes with a noncollinear spin configuration of adjacent magnetic layers of the tunnel junction under resonant excitation of spin vibrations due to current transfer of rotational moment in the presence of a bias current near and above the boundary of micromagnetic instability have the highest microwave sensitivity to microwave irradiation $[4,17,20-22]$. We calculated the resonance dependence of the microwave sensitivity of the spin diode based on the Landau - Lifshitz equations modified by Slonczewski, for several values of the bias current [20]. The calculated parameters are close to the parameters of the spin diode of [17], namely: the nanocolumn tunnel structure had a diameter of $D=140 \mathrm{~nm}$, the thickness of the free layer $d=2 \mathrm{~nm}$, the magnetization $M_{s}=$ $10^{6} \mathrm{~A} / \mathrm{m}$ and the magnetoresistance $\frac{\Delta R}{R}=130 \%, S \bar{R}=10 \mathrm{Ohm} \cdot \mu \mathrm{m}^{2}$, spin-polarization factor $\eta_{/ /}^{0}=0.63$, magnetic attenuation parameter $\alpha=0.01$.

The maximum microwave sensitivity occurs at the resonance frequency that depends on the effective magnitude of the magnetic field acting on the spins of the free layer. Maximum sensitivity falls when frequency increases. Table 3 shows the literature data on the microwave sensitivity of spin diodes, obtained from theoretical calculations and experimental measurements. It is apparent that the sensitivity of the spin diode can be comparable with the sensitivity of a Schottky semiconductor diode in the range up to $10 \mathrm{GHz}$. 
Table 2. Microwave sensitivity of the spin diode and its controlling parameters - frequency, external magnetic field, bias current.

\begin{tabular}{|l|l|l|l|l|l|}
\hline$\varepsilon, \mathrm{mV} / \mathrm{mW}$ & $\mathrm{f}, \mathrm{GHz}$ & $\mathrm{H}$, Oersted & $\mathrm{J}, \mathrm{A} / \mathrm{cm} 2$ & $\begin{array}{l}\text { Experiment/ } \\
\text { Theory }\end{array}$ & Link \\
\hline 12000 & 1.53 & 1100 & $3.8 \times 10^{-6}$ & experiment & {$[16]$} \\
\hline 970 & 1.2 & 0 & 0 & experiment & {$[17]$} \\
\hline 75000 & 1.1 & 0 & $2.7 \times 10^{-6}$ & experiment & {$[17]$} \\
\hline 30 & 13.5 & 0 & 0 & experiment & {$[4]$} \\
\hline 20000 & 9.2 & 500 & $7 \times 10^{-6}$ & theory & {$[20]$} \\
\hline 40 & 10 & 3200 & 0 & theory & {$[22]$} \\
\hline
\end{tabular}

From formulas (2) and (3), it follows that the detection range associated with microwave resolution increases with decreasing wavelength, and the power limited one decreases due to a decrease in the useful area of the emitting and receiving antenna element. In this regard, there is an optimal wavelength where the holographic detection range while using Schottky diodes as microwave receivers reaches maximum

$$
\lambda_{0}=\left(\frac{2 Y r_{n}}{\kappa}\right)^{\frac{1}{2}} \cdot\left(\frac{8 N P_{N}}{\pi \eta I_{s} R^{2}}\right)^{\frac{1}{4}} \sim 0.5-1 \mathrm{~cm}
$$

Spin diodes, as follows from Table 2, are close in microwave sensitivity to the sensitivity of Schottky diodes and close to optimal in the frequency range. Unlike a semiconductor measurement-base that includes an additional unit with a microwave vector spectrum analyzer for conducting fast and stable phase measurements, spin diodes can directly be used as nanoscale nonlinear elements for mixing signals, in combination with an RF synchronous amplifier for reading a rectified signal $[5,23]$. This contributes to the structural simplification and miniaturization of the holographic device.

\section{Conclusion}

The analysis of numerical estimates, numerical simulation results, and literature data shows that spin diodes can be promising for creating a holographic microwave-imaging device designed for autonomous driving of a vehicle in restricted traffic conditions with poor visibility and high noise. Their high microwave sensitivity and frequency range are close to the optimal parameters of microwave holographic imaging for these conditions. An acceptable recognition quality maintained up to $20 \mathrm{~m}$, which is in good agreement with the stated goals of detecting road obstacles at the near distance. The most safe reception conditions for a wavelength of $3 \mathrm{~cm}$ and a reception window of $2 \mathrm{~m}$ met with the number of receiving antenna sensors along the width of the reception window of at least 16. The optimal distance with respect to noise tolerance is up to $6 \mathrm{~m}$; noise resistance is quite high: a practically acceptable resistance level maintained up to $20 \mathrm{~m}$.

The Russian Science Foundation (project No. 19-12-00432) supported this work.

\section{References}

1. Holl P.M., Reinhard F., Holography of wi-fi radiation // Phys. Rev. Lett. 118, 183901 (2017).

2. Chetty K., Smith G.E., Woodbridge K., Through-the-wall sensing of personnel using passive bistatic wifi radar at standoff distances //IEEE Trans. on Geoscience and Remote Sensing 50(4), 1218-1226 (2012).

3. $\quad \mathrm{Fu} \mathrm{L}$. et al., Microwave holography using a magnetic tunnel junction based spintronic microwave sensor // J. Appl. Phys. 117(21), 213902 (2015).

4. Gui Y.S. et al., New horizons for microwave applications using spin caloritronics // Solid State Communications 198, 45-51 (2014).

5. Yao B.M. et al., Rapid microwave phase detection based on a solid state spintronic device // Appl. Phys. Lett. 104, 062408 (2014). 
6. Cao Z.X. et al., Spintronic microwave imaging // Appl. Phys. A 111(2), 329-337 (2013).

7. Speed, speed limits and stopping distances // Brake: the road safety charity. URL: http://www.brake.org.uk/fundraise/15-facts/1255-speed (retrieval date: May 05, 2020).

8. Gabor D., A new microscopic principle // Nature 161, 777-778 (1948).

9. Yamaguchi I., Zhang T., Phase-shifting digital holography // Optics letters 22(16), 12681270 (1997)

10. Kreis T., Handbook of holographic interferometry: optical and digital methods - John Wiley \& Sons, 2006.

11. Каленков Г.С., Каленков С.Г., Штанько А.Е., Гиперспектральная голографическая фурье-микроскопия // Квантовая электроника. - 2015. - Т. 45. - №. 4. - С. 333338. (Kalenkov G.S., Kalenkov, S.G., Shtan'ko A.E., Hyperspectral Holographic FourierMicroscopy // Quantum Electronics 45(4), 333-338 (2014) - in Russian).

12. Born M., Wolf E. Principles of Optics - Cambridge Univ. Pr., 1999.

13. Leshchiner D. et al., Resolution Limits in Near-Distance Microwave Holographic Imaging for Safer and More Autonomous Vehicles // J. of Traffic and Transportation Engineering 5, 316-327 (2017).

14. Leshchiner D. et al., Image reconstruction algorithms for the microwave holographic vision system with reliable gap detection at theoretical limits // EPJ Web Conf. 185 (2018).

15. Tikhonov A.N. et al., Numerical methods for the solution of ill-posed problems // Mathematics and Its Applications 328 - Springer Science \& Business Media, 1995.

16. Miwa S. et al., Highly sensitive nanoscale spin-torque diode // Nature Materials 13(1), 50-56 (2014).

17. Fang B. et al., Giant spin-torque diode sensitivity in the absence of bias magnetic field // Nature Communications 7, 11259 (2016).

18. Plokhov D.I. et al., Current-driven quantum dynamics of toroidal moment in rare-earth nanoclusters // EPJ Web Conf. 185 (2018).

19. Petrov P.N. et al., Inverse spin Hall effect in heterostructures "nanostructured ferromagnet/topological insulator" // EPJ Web Conf. 185 (2018).

20. Кулагин Н.Е. и др., Нелинейный токовый резонанс в спиновом диоде с плоскостным намагничиванием // Физика низких температур. - 2017. - Т. 43. - №. 6. - С. 889-897. (N.E. Kulagin et al., Nonlinear Current Resonance in the Spin-Torque Diode with a Planar Magnetization // Low Temperature Physics 43, 708 (2017) - in Russian).

21. Demin G., Popkov A., Spin-torque quantization and microwave sensitivity of a nanosized spin diode // EPJ Web Conf. 185 (2018).

22. Попков А.Ф. и др., Полевые особенности микроволновой чувствительности спинового диода при наличии тока смещения // Известия высших учебных заведений. Электроника. - 2017. - Т. 22. - №. 2. - C. 109-119. (Popkov, A.F et al., Field Features of Spin-Torque Diode Microwave Sensitivity Presence of Bias Current // Proceedings of Universities. Electronics 22, 109-119 (2017) - in Russian).

23. Hemour S. et al., Towards low-power high-efficiency RF and microwave energy harvesting // IEEE transactions on microwave theory and techniques 62(4), 965-976 (2014). 